\title{
Pour un avenir numérique de la communauté médicale
}

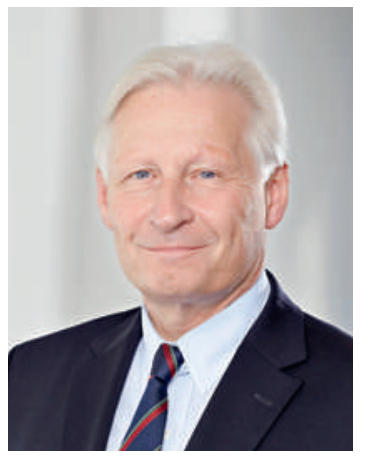

Dans toute la Suisse, les projets de cybersanté poussent comme des champignons. Tous poursuivent l'objectif avéré de sécuriser les échanges d'informations de patients entre les médecins, les hôpitaux, les cliniques et les autres institutions. Ils devraient simplifier les échanges, encourager l'efficacité et surtout augmenter la sécurité des patients tout au long des traitements. Quand on y regarde de plus près, on remarque cependant que toute la chaîne thérapeutique n'a pas pu être prise en considération, du moins jusqu'à présent, car bon nombre de projets ont été réalisés à l'initiative des cantons, des hôpitaux ou de groupements d'hôpitaux, et se sont concentrés sur la gestion informatique de certains documents seulement.
Si plusieurs cabinets voulaient créer ensemble une communauté, ils devraient investir doublement pour la mise en place de l'infrastructure externe, en plus des investissements informatiques habituels.

\section{Combler soi-même les trous béants}

Nous ne pouvons attendre plus longtemps qu'un quelconque projet-pilote s'empare du problème et comble les lacunes. En tant que libres praticiens, nous devons agir par nous-mêmes. Il s'agit de créer notre propre solution en termes d'infrastructure, qui réduise les coûts de chacun, permette d'accéder aux solutions de cybersanté existantes ou à venir, et garantisse la protection de toutes les données des patients, ainsi que le prévoient la loi sur le dossier électronique du patient et la stratégie en matière de cybersanté de la Confédération. C'est l'objectif que s'est fixé la FMH en tant qu'association professionnelle.

Pour éviter une dépendance envers les fournisseurs externes, l'infrastructure doit être une solution de médecin à

\section{Nous devons réaliser notre propre solution d'infrastructure qui réduit les coûts de chaque médecin et permet le raccordement aux solutions de cybersanté.}

\begin{abstract}
Coûts d'investissement élevés et nouvelles attentes
A cela s'ajoute une difficulté supplémentaire pour les médecins en pratique privée. S'ils exercent dans un cabinet de groupe ou un cabinet individuel, ils sont rarement reliés à l'infrastructure informatique globale d'une institution plus grande. En fait, il ne serait pas si simple de les intégrer dans un réseau sécurisé existant. Construire leur propre infrastructure sécurisée demanderait du temps et coûterait cher, ce ne serait pas judicieux pour beaucoup et pas vraiment rentable.
\end{abstract}

\section{Avec son système d'identification et de communi- cation sécurisée, l'infrastructure HIN est le point de départ idéal pour atteindre l'objectif de la FMH de créer une plateforme pour les médecins.}

C'est pourquoi de nombreux projets de cybersanté ont évité d'intégrer les libres praticiens qui, en plus des échanges d'informations via les interfaces entre cabinets et hôpitaux, ont d'importantes attentes en matière de communication, comme l'échange d'informations pertinentes pour les traitements, dans l'idée d'une intégration horizontale et verticale. médecin. A cet égard, la communauté HIN constitue un excellent point de départ. La plateforme dispose en effet déjà d'un système de communication sécurisé. Le noyau de celleci est constitué de l'infrastructure HIN et de ses prestations en matière d'identification, de communication sécurisée et d'interaction dans le réseau. Il peut être développé avec très peu d'obstacles technologiques pour devenir une infrastructure de la communauté des médecins en libre pratique et être facilement utilisable grâce au système d'identification HIN. En posant ainsi les jalons d'une infrastructure de la communauté des médecins libres praticiens, HIN deviendra une plateforme sur laquelle d'autres communautés pourront également venir se greffer et ainsi grandir.

Nous nous considérons comme un «Hub» pour les médecins installés, auquel tous les partenaires pourraient se connecter. Les prémisses les plus importantes doivent cependant rester la protection des données des patients et le respect du secret médical. Pour que les médecins en cabinet privé puissent également prendre part au monde numérique de demain.

Dr Urs Stoffel, membre du Comité central de la FMH, responsable du département eHealth infrastructure de sécurité et collecte des données 